\title{
Analytical method development and validation for the simultaneous estimation of lamivudine and tenofovir disoproxil fumarate by RP-HPLC method
}

\begin{abstract}
A simple, accurate, precise and sensitive reverse phase high performance liquid chromatography (RP-HPLC) method has been developed, which can separate and quantitatively estimate Lamivudine and Tenofovir disoproxil fumarate in mixed dosage form. The chromatographic separation for Lamivudine and Tenofovir disoproxil fumarate was achieved with mobile phase containing acetonitrile and phosphate buffer PH $3.5(80: 20 \% \mathrm{v} / \mathrm{v})$, reverse phase Thermo scientificTM HypersilTM BDS $5 \mu \mathrm{C} 18120 \mathrm{~A}(250 \times 4.60 \mathrm{~mm}$ i.d $)$ column in isocratic mode at room temperature and UV detection at $260 \mathrm{~nm}$. The compounds were eluted at a flow rate of $1.2 \mathrm{ml} / \mathrm{min}$. The retention times of Lamivudine and Tenofovir disoproxil fumarate were found to be $2.33 \mathrm{~min}$ and $2.68 \mathrm{~min}$ respectively. The above method was validated in terms of system suitability, linearity, accuracy, precision, Limit of Detection (LOD), Limit of Quantification (LOQ) in accordance with ICH guidelines. The method was rapid, simple, economical and suitable for routine quality control analysis.
\end{abstract}

Keywords: lamivudine, tenofovir disoproxil fumarate, analytical method development, validation, RP-HPLC
Volume 4 Issue 5 - 2016

Anil Dubbaka, Sireesha D, Vasudha Bakshi

Department of Pharmacy, Anurag group of Institutions, India

Correspondence: Sireesha D, Department of Pharmacy, Anurag group of Institutions (Formerly Lalitha College of Pharmacy), Hyderabad-500088, India, Email sireeshapharmacy@cvsr.ac.in

Received: October 26, 2016 | Published: November 30, 2016

\section{Introduction}

Lamivudine(LAM)(4-amino-1-[(2R,5S)-2-(hydroxymethyl)1,3-oxathiolan-5-yl]-1,2-dihydropyrimidin-2-one) is nucleoside analogues with a structure that consists of a pyrimidine base, which is $\mathrm{N}$-substituted at the 1-position with a 3'-thia derivative (1,3-oxazolidine) of the ribose moiety that is characteristic of nucleosides. It is reverse trascriptase inhibitor and zalcitabine analog in which a sulfur atom replaces the 3' carbon of the pentose ring. It is used to treat Human Immunodeficiency Virus Type 1 (HIV-1) and hepatitis B (HBV). This compound belongs to the class of organic compounds known as 3'-thia pyrimidine nucleosides.

Tenofovir disoproxil fumarate(TDF) (a prodrug of tenofovir) ( $\{[(2 \mathrm{R})-1-(6$-amino-9H-purin-9-yl)propan-2-yl]oxy $\}$ methyl) phosphonic acid), belongs to a class of antiretroviral drugs known as nucleotide analogue reverse transcriptase inhibitors (nRTIs), which block reverse transcriptase, an enzyme crucial to viral production in HIV-infected people. In vivo tenofovir disoproxil fumarate is converted to tenofovir, an acyclic nucleoside phosphonate (nucleotide) analog of adenosine 5 '-monophosphate. ${ }^{2}$

Both the drugs are marketed as combined dose tablet formulation in the ratio of 300:300 mg LAM: TNF. Literature survey revealed that there is no method reported for the simultaneous estimation of these drugs, some methods for estimation of individual drugs or with other drugs UV-spectrophotometry, ${ }^{3-8}$ RP-HPLC ${ }^{9}$ are available. HPLC method is more sensitive compared to UV; therefore, there is a challenge to develop RP-HPLC method for the simultaneous estimation of Lamivudine and Tenofovir disoproxil fumarate. The present study was involved in a research effort aimed at developing and validating a simple, accurate, precise and economical RP-HPLC method for the simultaneous estimation of Lamivudine and Tenofovir disoproxil fumarate mixed dosage form.

\section{Materials and methods}

Acetonitrile (HPLC grade) and Water (HPLC grade) were obtained from Merck specialties private limited, Mumbai, India. Orthophosphoric acid (GR grade) was obtained from S.D. Fine Chemicals Ltd, Mumbai, India. Potassium Dihydrozen orthophosphate (KH2PO4) was obtained from Merck specialties private limited Mumbai, Dipotassium hydrogen phosphate (K2HPO4) S.D Fine Chemical ltd. Mumbai. Pure drugs of Lamivudine and Tenofovir disoproxil fumarate were obtained as gift samples from Hetero labs (Hyderabad, India). TENOLAM tablets manufactured by Hetero Pharmaceutical Limited purchased from local pharmacy are used for the analysis. The label claim states that this formulation contains $300 \mathrm{mg}$ of Lamivudine and 300mg of Tenofovir disoproxil fumarate.

\section{Instrumentation and chromatographic condition}

HPLC method development and validation were done on SHIMADZU (Japan) liquid chromatography equipped with LC-20AD pump, LC 20A UV/Vis detector and Rheodyne 7725i injection with a $20 \mu \mathrm{L}$ loop. For instrument control, data acquisition and processing, the chromatographic system was interfaced to LC solutions software. Other instruments included are Shimadzu electronic balance BL220H (SHIMADZU corporation, Japan), Value 1 stage vacuum pump Model: VE115, Fast clean ultrasonic cleaner. The column used for chromatographic separations was reverse phase Thermo ScientificTM HypersilTM BDS $5 \mu$ C18 120A $(250 \times 4.60 \mathrm{~mm}$ i.d $)$. The analytical wave length was set as $232 \mathrm{~nm}$ and samples of $20 \mu \mathrm{L}$ were injected. The chromatographic separations were accomplished using mobile phase consisting of Acetonitrile: phosphate buffer PH-3.5 (80:20\%v/v), filtered through $0.45 \mu \mathrm{m}$ filter using Value 1 stage vacuum pump and desecrated in fast clean ultrasonic cleaner. Mobile phase was pumped in isocratic mode at a flow rate of $1.2 \mathrm{ml} / \mathrm{min}$ at room temperature (Figure $1 \& 2$ ). 
<smiles>Nc1ccn([C@@H]2CS[C@H](CO)O2)c(=O)n1</smiles>

Figure I Structure of Lamivudine.

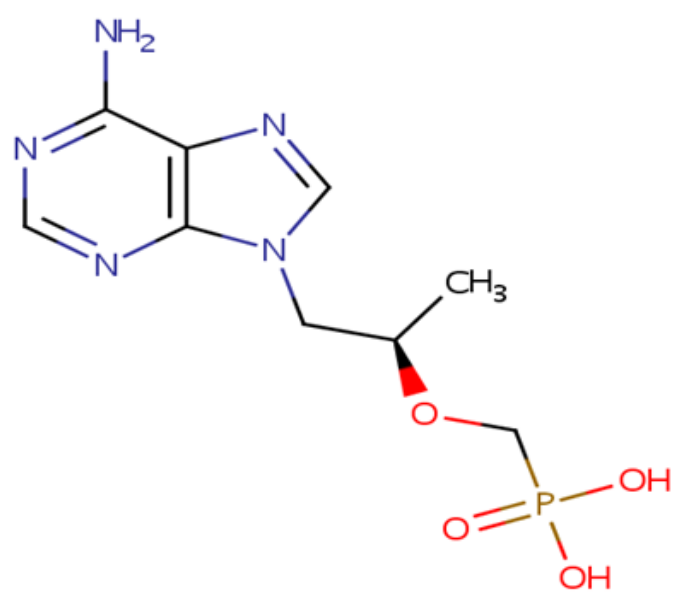

Figure 2 Structure of Tenofovir disoproxil fumarate.

\section{Preparation of standard solutions}

Stock solutions were prepared by dissolving $10 \mathrm{mg}$ of Lamivudine and $10 \mathrm{mg}$ of Tenofovir disoproxil fumarate, in $10 \mathrm{ml}$ of acetonitrile separately. Aliquots of the standard stock solutions of Lamivudine and Tenofovir disoproxil fumarate were transferred into $10 \mathrm{ml}$ volumetric flasks and solution was made up to the volume to yield required concentrations of Lamivudine and Tenofovir disoproxil fumarate. A typical chromatogram obtained from the analysis of drugs using the developed method is shown in Figure 3.

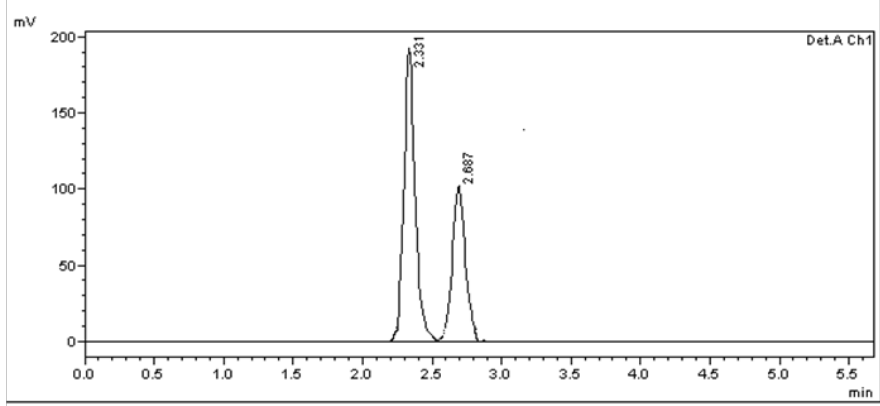

Figure 3 Chromatogram of standard solution $(10 \mu \mathrm{g} / \mathrm{ml}$ Lamivudine and $10 \mu g / \mathrm{ml}$ Tenofovir).

\section{Preparation of sample solution for assay}

Ten TENOLAM tablets each containing $300 \mathrm{mg}$ of Lamivudine and $300 \mathrm{mg}$ of Tenofovir disoproxil fumarate were weighed, average weight was calculated and powdered. A quantity equivalent to $100 \mathrm{mg}$ of Lamivudine and $100 \mathrm{mg}$ of Tenofovir disoproxil fumarate was weighed and transferred into $10 \mathrm{ml}$ volumetric flask. It was extracted with acetonitrile. The volumetric flask was sonicated for 5minutes to affect the complete dissolution of the drugs and the solution was made up to the volume with methanol and filtered. Suitable aliquots of formulation solution were prepared and injected to HPLC to obtain concentration in the linearity range (Figure 4).

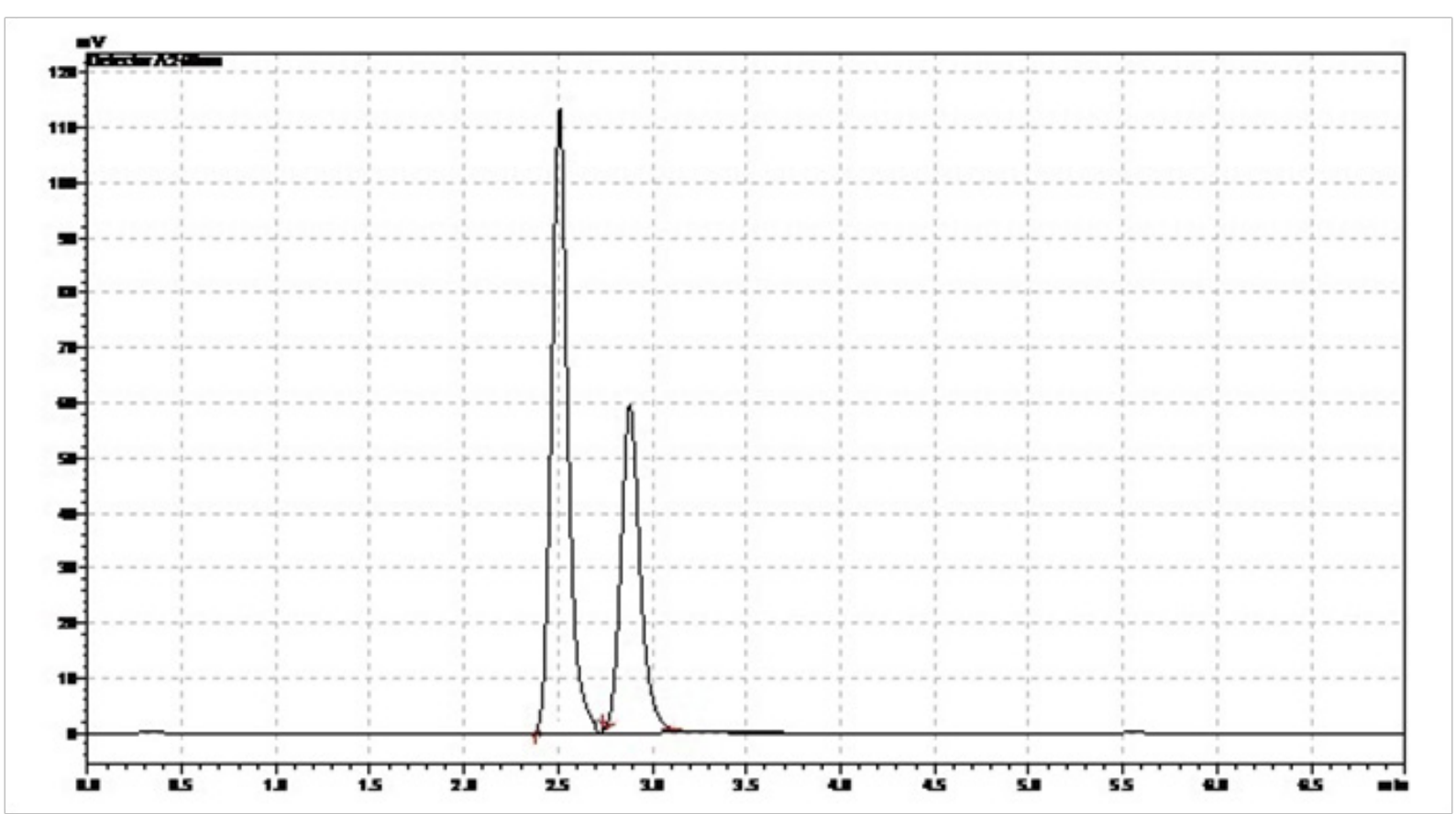

Figure 4 Chromatogram of formulation ( $10 \mu \mathrm{g} / \mathrm{ml}$ Lamivudine and $\mathrm{I} 0 \mu \mathrm{g} / \mathrm{ml}$ Tenofovir) 


\section{Results and discussion}

\section{Method Development}

For the RP-HPLC, chromatographic conditions were optimized to get best resolution and peak shape. The selection of mobile phase was based on peak parameters like symmetry, theoretical plates and capacity factor. Symmetrical peaks with good separation (retention time for Lamivudine is 2.331 and Tenofovir disoproxil fumarate is 2.687) were obtained with reverse phase Thermo ScientificTM HypersilTMBDS $5 \mu \mathrm{C} 18120 \mathrm{~A}(250 \times 4.60 \mathrm{~mm}$ i.d $)$ column. The mobile phase containing Acetonitrile: phosphate buffer PH-3.5(80:20\% v/v) was used at a flow rate of $1.2 \mathrm{ml} / \mathrm{min}$. The optimum wavelength for detection and quantification was at $260 \mathrm{~nm}$, at which good detector response was obtained for both the results are given in Table 1 .

Table I Summary of validation parameters

\begin{tabular}{lll}
\hline Parameters & Lamivudine & $\begin{array}{l}\text { Tenofovir disoproxil } \\
\text { fumarate }\end{array}$ \\
\hline Linearity range $(\mu \mathrm{g} / \mathrm{ml})$ & $15-35$ & $15-35$ \\
Correlation coefficient & 0.999 & 0.999 \\
Slope & 41816 & $3055 \mathrm{I}$ \\
Intercept & 26084 & 4169 \\
LOD $(\mu \mathrm{g} / \mathrm{ml})$ & 0.4632 & 1.46 \\
LOQ $(\mu \mathrm{g} / \mathrm{ml})$ & 1.544 & 4.29 \\
Recovery $(\%)$ & $99.11 \%$ & $99.62 \%$ \\
80 & $99 \%$ & $99.33 \%$ \\
I00 & $99.39 \%$ & $99.79 \%$ \\
I 20 & 1.05 & 0.721 \\
Precision $(\mathrm{RSD} \%)$ & 1.063 & 0.942 \\
Intraday $(\mathrm{n}=6)$ & 3369 & 3389 \\
Interday $(\mathrm{n}=6)$ & 1.22 & 1.23 \\
Theoretical plates & 2.6 & \\
Tailing factor & & \\
Resolution & & \\
\end{tabular}

\section{Method validation}

As per ICH guidelines, the method validation parameters checked were linearity, precision, accuracy, limit of detection and limit of quantification. ${ }^{10}$

\section{Linearity and range}

Linearity was established by least squares linear regression analysis of the calibration curve. The calibration curves were linear over the concentration range of $15-35 \mu \mathrm{g} / \mathrm{ml}$ for Lamivudine and $15-$ $35 \mu \mathrm{g} / \mathrm{ml}$ for Tenofovir disoproxil fumarate. Peak areas were plotted versus respective concentrations and linear regression analysis was performed on the resultant curves. Correlation coefficients were found to be 0.999 and 0.999 for Lamivudine and Tenofovir disoproxil fumarate respectively (Figure $5 \& 6$ ). The results are given in Table 1 .

\section{Precision}

The precision of the analytical method was studied by multiple sampling of the homogenous sample. The precision was done at two levels (intraday and inter day). Intraday precision was done by analyzing the intermediate concentration of each drug for six times. Interday precision was measured over three consecutive days for the same drug concentrations for six times. The \%RSD values were found to be calculated for each of them and the low RSD values indicate that the method is precise. The results are given in Table 1.

\section{Accuracy}

Recovery studies were carried out by applying the method to drug sample to which known amount of standard Lamivudine and Tenofovir disoproxil fumarate corresponding to $80 \%, 100 \%$ and $120 \%$ of label claim had been added. At each level of the amount three determinations were performed. The results are given in Table 1.

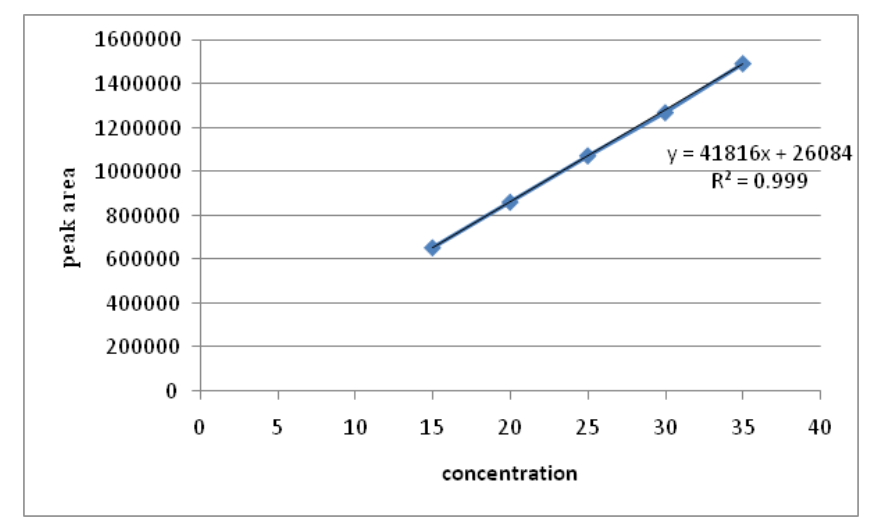

Figure 5 Calibration graph of Lamivudine.

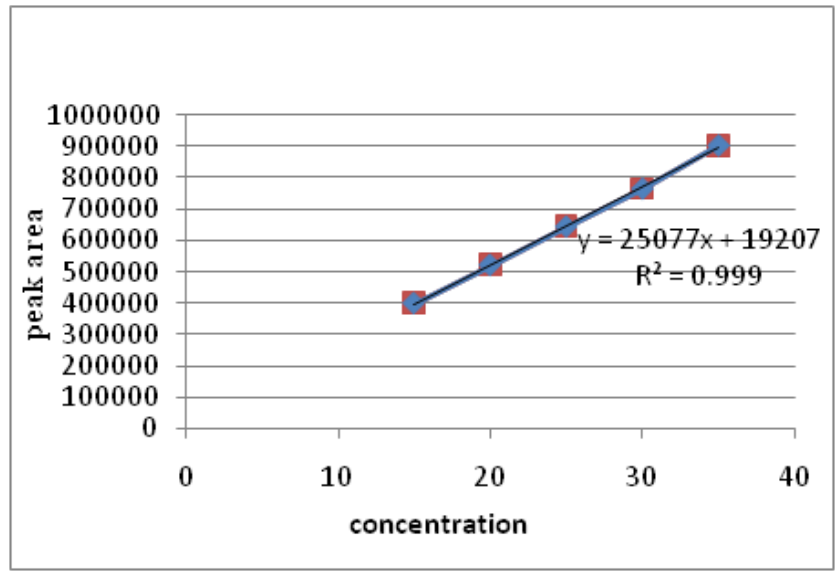

Figure 6 Calibration graph of Tenofovir by RP-HPLC.

\section{Sensitivity}

LOD and LOQ decide about the sensitivity of the method. LOD is the lowest detectable concentration of the analyte by the method while LOQ is the minimum quantifiable concentration. LOD and LOQ were calculated by standard calibration curves. The results are given in Table 1 .

\section{System suitability studies}

System suitability parameters like number of theoretical plates (N), peak asymmetry factor (As), tailing factor etc., were studied. The results are given in Table 1.

\section{Analysis of marketed formulation}

The proposed procedures were successfully applied for the simultaneous estimation of Lamivudine and Tenofovir disoproxil fumarate in the formulation and the drug contents in each sample were calculated by comparison with the appropriate standard solution of the drug. The results obtained were in agreement with label claim. The results of analysis are given in (Table 2) (Figure 4). ${ }^{11-13}$ 
Table 2 Analysis of marketed formulation

\begin{tabular}{lllll}
\hline Drug & Amount labeled $(\mathbf{m g})$ & Amount found(mg) & \%Assay & \%RSD* \\
\hline Lamivudine & 300 & 299.02 & 99.67 & 0.65 \\
Tenofovir & 300 & 301.13 & 100.3 & 0.715 \\
\hline
\end{tabular}

*Mean of three observations

\section{Conclusion}

RP-HPLC methods enable the quantitation of Lamivudine and Tenofovir disoproxil fumarate in oral dosage form with good accuracy and precision, either in laboratory prepared samples or in pharmaceutical dosage forms. The good recoveries were obtained in all cases as well as the reliable agreement with the reported procedure proved that the proposed methods could be applied efficiently for determination of Lamivudine and Tenofovir disoproxil fumarate in oral dosage form with satisfactory precision. This method is considered simple, reliable, economical providing satisfactory accuracy, precision with lower limits of detection and quantification more sensitive. More over the shorter duration of analysis for Lamivudine and Tenofovir disoproxil fumarate makes the reported method suitable for routine analysis in mixed dosage form.

\section{Acknowledgements}

I am very thankful to Anurag group of institution (Formerly Lalitha college of pharmacy), Hyderabad. For giving permission to carry out my work.

\section{Conflict of interest}

The author declares no conflict of interest.

\section{References}

1. http://www.drugbank.ca/drugs/DB01132

2. http://www.drugbank.ca/drugs/DB00222

3. Bhargavi S, Suryasagar G, Sowmya KD, et al. UV Spectrophotometric Method for Determination of Lamivudine in Pharmaceutical Dosage Forms. Int J Pharm Sci Rev Res. 2013;21(2):131-133.

4. Anandakumar Karunakaran, Kannan Kamarajan, Vetrichelvan Thangarasu. A Validated RP-HPLC method for simultaneous estimation of Lamivudine and Tenofovir disoproxil fumarate in pure and in tablet dosage form. Eurasian J Anal Chem. 2012;7(2):56-66.
5. Devmurari P. Simultaneous Spectrophotometric Determination of Lamivudine and Abacavir in the Mixture. Int J Pharm Sci and Res. 2010;1(7):82-86.

6. dube D, Vyas SP. Simultaneous Spectrophotometric Estimation of Lamivudine and Silymarin in Mixture. Int J Pharmacy and Pharm Sci. 2009;1(2):107-111.

7. Soboleva E, Ambrus A. Application of system suitability test for quality assurance and performance optimization of a gas chromatographic system for pesticide residue analysis. J Chromatogr A . 2004;1027:55-65.

8. Kano EK, dos Reis Serra CH, Koono EE, et al. Determination of Lamivudine in human plasma by HPLC and its use in bioequivalence studies. J Pharm Biomed Anal. 2006;4(3):761-765.

9. Bahrami G, Mirzaeei S, Kiani A, et al. High-Performance Liquid Chromatographic determination of Lamivudine in human serum using liquid-liquid extraction; application to pharmacokinetic studies. $J$ Chromatogr B. 2005;823(2):213-217.

10. Gomes NA, Vaidya VV, Pudage A, et al. Liquid ChromatographyTandem Mass Spectrometry (LC-MS/MS) method for simultaneous determination of Tenofovir and Emtricitabine in human plasma and its application to a bioequivalence study. J Pharm Biomed Anal. 2008;48(3):918-926.

11. Basavaiah K, Somashekar B C, Ramakrishna V. Rapid titrimetric and spectrophotometric assay methods for the determination of Lamivudine in Pharmaceuticals using iodate and two dyes. J Anal Chem. 2007;62(6):542-548

12. Abdool Karim Q, Abdool Karim SS, Frohlich JA, et al. Effectiveness and Safety of Tenofovir Gel, an Antiretroviral Microbicide, for the Prevention of HIV Infection in Women. Science. 2010;329(5996):11681174.

13. British Pharmacopoeia. British Pharmacopoeia 2009. The Stationary Office, British Pharmacopoeia Commission, 2009. 